\title{
La politique publique de la gestion des espaces verts par l'hôtel de ville de Kinshasa
}

\author{
Jules Kassay Ngur-Ikone*
}

\section{Résumé}

Sur le plan urbanistique de la ville de Kinshasa (capitale de la RDC), les colons, avaient prévu les espaces verts pour un cadre de vie urbain, verdoyant et permettant de se détendre et embellir la ville. De 1960 à 1980, la même politique publique urbanistique a été respectée. Mais actuellement, avec l'explosion démographique, les espaces verts qui, jadis, faisaient la fierté de la capitale de la RDC dans chaque commune, ont été les uns effacés de la carte géographique et les autres spoliés par les habitats précaires sous prétexte qu'il faut diminuer l'insalubrité et l'insécurité. Il y a également la présence petits métiers et des marchés spontanés qui favorisent l'insalubrité. Et tout ce théâtre se produit sous l'œil impuissant du gouverneur de la ville.

Notre préoccupation est de démontrer l'importance des espaces verts dans le paysage d'une ville, faire le bilan de la gestion de ces espaces par la population bénéficiaire de cette œuvre et par l'autorité urbaine et enfin proposer des perspectives.

\begin{abstract}
In terms of the urban planning of the city of Kinshasa (capital of the DRC), the colonial authorities had planned to have green spaces to provide the urban living space with a greenery that would promote relaxation and beautify the city. The same public urban development policy continued to be applied from 1960 to 1980; but nowadays, with the population explosion, the green spaces that used to be the pride of the Congolese capital either wiped off the map or spoilt by slum dwellings on the pretext of reducing insalubrity and insecurity.
\end{abstract}

\footnotetext{
* Département des Sciences Politiques et Administratives, Centre d’Etudes Politiques (CEP) Université de Kinshasa, R.D.Congo.

E-mail : jkassay2000@yahoo.fr
} 
The presence of small trades and spontaneous markets also promotes insalubrity. And it is all taking place under the helpless eyes of the governor of the city.

Our aim is to demonstrate the importance of green spaces in the city landscape, to review the management of these spaces by the beneficiary population and by the urban authorities, and finally to propose a way forward.

\section{Introduction}

Cela fait exactement cinquante ans (1960-2010) que la République Démocratique du Congo a accédé à l'indépendance. Sur le plan urbanistique de la ville de Kinshasa (capitale de la RDC), le colonisateur avait prévu les espaces verts pour un cadre de vie urbain, verdoyant et permettant de se détendre et d'embellir la ville avec des espèces d'arbres de la forêt équatoriale et importés. De 1960 à 1975, la même politique publique urbanistique était respectée à la lettre. En effet, de 10987 habitants, à la création de la ville de Kinshasa, la population kinoise est passée de 404620 en 1960 à 6062000 habitants en 2000 (Le Potentiel 2005) Les dernières estimations, selon le recensement administratif de 2005, sont à 7500000 habitants. Cette population, estime-t-on, pourrait atteindre plus de 10 millions d'habitants en 2015, se hissant ainsi parmi les 30 plus grosses agglomérations mondiales (United Nations 2007).

De ce fait, la ville de Kinshasa concentre du coup tous les problèmes qu'on retrouve dans des grandes villes du Tiers-Monde, en particulier la défaillance du système de gestion de l'environnement urbain, qui se traduit, entre autres, par une diversité de pollutions urbaines. Singulièrement, ces pollutions sont très marquées dans les quartiers et communes populaires (Kingasani, Malweka, Kingabwa, Kisenso, Selembao, Makala, ...) à forte densité de concentration humaine et dans des foyers économiques du type des marchés, où se concentre à longueur de journée, même jusque tard la nuit, une multitude de négociants tant formels qu'informels, avec tout ce que cela peut entraîner comme conséquences sur le plan de la dégradation de l'environnement urbain.

Les espaces verts dans les villes africaines faisaient jadis la fierté. Par exemple, la ville de Brazzaville (République du Congo) était surnommée « la verte », parce qu'elle était aménagée par une floristique des différentes espèces d'arbres (cour ou jardin, clôture et plantation intra-urbaine). Malheureusement, ces espaces verts couvrent aujourd'hui des nids-de-poule. De son histoire, la ville de Lomé et les espaces verts restent inséparables, déjà la traduction du nom du premier village "Alomé » est significative. C'est la localité située dans les buissons d'Alo. Aujourd'hui, les buisons de la ville de Lomé sont remplacés par la saleté (Actes de colloque de Lomé 
1997:233-238) Kinshasa, la capitale de la RDC, hier « Kin-kiese » (Kin-la joie), a cédé son sourire à la tristesse. Les espaces verts de cette capitale ont été effacés de la carte géographique du fait de leur spoliation, au profit des habitats précaires, sous prétexte d'éliminer l'insalubrité et l'insécurité (le cas de spoliation de l'espace vert de Limete Socopao, quartier sans-fil, ...), d'autres ont été remplacés par de petits métiers et des marchés pirates, avec leur cortège de déchets quotidiens. Et tous ces théâtres se produisent sous l'œil impuissant des différents locateurs de l'Hôtel de ville, des bourgmestres et des chefs des quartiers qui sont sensés appliquer les lois en la matière.

En outre, ces autorités ne donnent pas de signes qui montrent qu'il est aujourd'hui difficile de s'intéresser à la gestion urbaine des espaces verts et aux questions de l'environnement urbain sans faire référence au développement durable. C'est une préoccupation majeure depuis quelques décennies pour les villes en général et celles africaines en particulier. Dans le processus de développement de ces villes, entamé depuis la colonisation (et anté-rieurement pour certaines d'entre elles), les politiques publiques urbaines en matière d'espaces verts et de protection de l'environnement ne coïncident pas toujours avec les pratiques de la plupart des citadins. Loin d'être hostiles à l'action publique, les citadins marquent pour la plupart une indifférence face aux services et entreprises d'assainissement de la ville qui sont menés par l'Hôtel de ville de Kinshasa et ses partenaires (les opérateurs privés et les associations qui s'occupent de cette gestion). Les efforts que ces multiples intervenants déploient en matière de gestion des espaces verts ne semblent pas contribuer à modifier les pratiques de certains citadins qui se caractérisent par une certaine désinvolture dans la façon de disposer un espace public (United Nations 2007). Notre préoccupation, dans ce papier, est de démontrer l'importance des espaces verts dans le paysage d'une ville, de faire le bilan de la gestion de ces espaces par la population bénéficiaire de cette œuvre et par l'autorité urbaine. Il s'agira aussi d'esquisser quelques perspectives d'avenir dans le domaine de la gestion des espaces verts. Cela étant, notre étude est subdivisée en quatre axes. La première porte sur quelques considérations d'ordre conceptuel et théorique, le deuxième axe est consacré à un bref historique de la ville de Kinshasa, en rapport avec la gestion des espaces verts. Le troisième axe rend compte de l'état des lieux des espaces verts dans la ville de Kinshasa et le dernier axe aborde les conséquences déterminantes de la destruction de la spoliation des espaces verts à Kinshasa, pour ressortir cette complicité qui existe entre l'espace public et l'espace vert. 


\section{Aspects théoriques et conceptuels}

\section{Cadre théorique et conceptuel}

\section{La Politique publique}

En parlant de la genèse de ce concept, deux questions fondamentales se posent. La première est celle de l'origine intellectuelle de l'analyse des politiques comme méthode pour étudier et comprendre l'action de l'État. La seconde question reste celle de l'origine des politiques publiques elles-mêmes. Elle consiste à se demander quelles transformations dans la société a pu produire cette explosion des régulations étatiques dont l'analyse des politiques cherche à rendre compte.

En effet, nous allons orienter l'explication de ce concept dans le sens du développement des interventions de l'État dans la société, non seulement dans l'Administration, mais aussi dans ses multiples domaines techniques utilisés par les décideurs, en l'occurrence la gestion des espaces verts.

Le concept "politique publique » est d'origine anglo-saxonne. Selon Kanoepfel et al, une politique publique constitue un enchaînement de décisions ou d'activités, intentionnellement cohérentes, prises par différents acteurs, publics et parfois privés, dont les ressources, les attaches institutionnelles et les intérêts varient, en vue de résoudre de manière ciblée un problème défini politiquement comme collectif. Cet ensemble de décisions et d'activités donne lieu à des actes formalisés, de nature plus ou moins contraignants, visant à modifier le comportement de groupes sociaux supposés à l'origine du problème collectif à résoudre (groupes cibles), dans l'intérêt de groupes sociaux qui subissent les effets négatifs dudit problème (bénéficiaires finaux) (Kanoepfel, Larrue et Varone, cités par Nahrath S. 2005).

La création, l'entretien et la gestion des espaces verts qui constituent des lieux publics importants dans la configuration générale des espaces publics nécessitent, sans nul doute, la mise en route des politiques publiques liées de manière générale à la gestion de l'environnement. Ces politiques publiques peuvent être prises au niveau national ou à l'échelle locale : celle des entités administratives décentralisées.

L'État national ou les États locaux sont donc appelés à intervenir dans le registre de la gestion des espaces verts à travers des services étatiques spécialisés. Les politiques publiques ayant trait à la gestion de ces espaces ont une forte dimension territoriale et urbanistique. Le caractère «territorialisé », "physique » ou "visible » de ces politiques est tel que toute défaillance en matière de gestion des espaces verts est vite perceptible parce qu'elle a un impact visible et sévère sur le territoire d'une ville ou sur celui d'une province. 


\section{Espace vert}

La notion d'espace vert appartient au vocabulaire de la planification urbaine et paysagère comme à celle de l'urbanisme paysager. Dans les agglomérations urbaines, l'espace vert désigne des terrains non encore bâtis, végétalisés ou arborés, boisés ou agricoles. A cet effet, les espaces verts sont souvent perçus comme des réserves foncières et donc des terrains à développer quand l'opportunité se présente.

C’est un espace planté ou garni de végétations. Mais précisons que tout espace planté ou garni de végétations ne doit pas nécessairement être compté comme espace vert.

Peut être compté considére espace vert, un jardin ou un espace planté, comportant en majorité des végétaux permanents, notamment des arbres de haute tige (Soulier 1968 :50). Cela n’est pas forcément vrai dans la mesure où un espace vert peut contenir aussi les arbres de tiges courtes ou longues de taille.

L'espace vert est un milieu de détente et de récréation, un lieu de promenade et de découverte de la nature ; il est un équipement public/privé très prisé des citadins. Au-delà de leur rôle social, les parcs, les squares, les jardins privés sont des espaces gérés et entretenus qui composent le « grain de verdure » d'une ville. Certes, les espaces verts sont aussi des milieux remaniés dont l'environnement et les pratiques culturales modifient les équilibres naturels.

\section{Genèse et définition de l'espace public}

Agora, espace public réel

L'espace public est un concept opératoire en histoire grecque. L'agora ${ }^{1}$ est, ainsi, le premier espace public réel de la culture politique grecque. En effet, dans la cité grecque antique, ce lieu désignait la place où se rencontraient les citoyens pour échanger leurs opinions sur la vie publique, pour se consacrer, entre eux, à leurs affaires et à la vie économique de la cité, pour pratiquer la sociabilité dont ils étaient porteurs. L'agora est, la place publique, le lieu de toutes les rencontres et le lieu où s'exerce la vie sociale. C'est pourquoi elle a fini par constituer un symbole de la cité tout entière : en effet, comme c'est dans ce lieu que se déroulaient les activités d'échanges et de communication par lesquelles les citoyens mettaient en pratique leur appartenance. Il a fini par servir de représentation à la cité elle-même.

\section{L'agora}

L'agora est devenue le signifiant de la vie sociale et politique constitutive de la cité antique. 
C'est que la vie sociale, dans la cité grecque se confond avec la vie politique. En effet, c'est dans leurs activités de rencontre des autres et de discussion - voire de confirmation - avec eux que les citoyens investissaient l'agora en faisant le lieu du débat politique. Comme l'agora, lieu de sociabilité collective, était aussi le lieu où se réunissait l'assemblée, l'ecclésia, elle s'est institutionnalisée comme lieu de la délibération et de la décision. C’est là que sont prises les décisions et que sont exercés les pouvoirs constitutifs de la cité et, par conséquent, elle est devenue le lieu dans lequel sont perçues l'appartenance et la sociabilité, par le passage à l'acte des réalités de la vie sociale.

\section{Espace public}

L'espace public fait aujourd'hui l'objet de sollicitations nombreuses et pressantes. C'est le lieu d'innombrables pratiques de la vie urbaine : commerce, détente, rencontre, etc. Ici, l'espace public exprime son rôle social, politique et économique. C'est aussi une vitrine où la ville forge son image et son identité. C'est encore l'endroit où s'exercent les fonctionnalités de la ville : déplacements, réseaux techniques, etc.

Au regard de ces enjeux extrêmement divers et importants, les difficultés des villes sont multiples puisqu'elles doivent faire face :

- à la multiplicité des intervenants - maîtrise d’ouvrage partagée, services municipaux, concessionnaires de réseaux, propriétaires, riverains, afficheurs, ... ;

- à la diversité des sites - centres anciens, secteurs périurbains, grands ensembles, ... ;

- aux conflits entre les différents usages dont l'espace public est la scène - déplacements, stationnement, commerce, emploi, détente, ... ;

- à la diversité des matériaux disponibles pour les revêtements de sols et les équipements, et à la prolifération de la signalisation, des insignes, des affiches et du mobilier urbain.

Aujourd'hui, l'évolution plus citoyenne des esprits et la prise de conscience des citadins en matière de qualité du cadre de vie incitent à des démarches plus qualitatives et à une plus grande exigence. La conception des espaces publics doit maintenant concilier la sécurité, l'accessibilité, le confort, l'esthétique, etc.

A cet effet, le concept d'espace public est aujourd'hui appliqué à des domaines très différents : non seulement à l'étude des rapports entre État et société, à la théorie de la démocratie et à l'analyse de l'action politique, ou encore à l'analyse des médias, mais aussi à l'étude de la construction sociale 
des problèmes publics, à celle des comportements et des relations en public, dans l'espace urbain en particulier, à l'enquête sur les scènes publiques urbaines et, plus largement, à l'approche environnementale de la vie sociale.

Alain Letourneau résume ainsi l'ouvrage dans lequel Habermas introduit en 1962 la notion d'espace public :

l'espace public, c'est un ensemble de personnes privées rassemblées pour discuter des questions d'intérêt commun. Cette idée prend naissance dans l'Europe moderne, dans la constitution des espaces publics bourgeois qui interviennent comme contrepoids des pouvoirs absolutistes. Ces espaces ont pour but de médiatiser la société et l’État, en tenant l’État responsable devant la société par la publicité, Öffentlichkeit dont parlait Kant. La publicité critique suppose d'obtenir l'information requise sur le fonctionnement de l’État, afin que celui-ci puisse être examiné et critiqué sous l'œil de l'opinion publique (Brunet 2001:49).

La conception abstraite de la démocratie telle que théorisée par Habermas et ayant trait à la question de la démocratie ne nous intéresse pas dans cette étude. C'est plutôt l'espace public dans la dimension territorialisée qui est mise en relief ici. L'espace public vu sous cet angle signifie lieu public, c'est-à-dire un espace territorial ouvert au public, à tous les citoyens et dont l'usage est réglementé par les pouvoirs publics. A ce titre, l'espace vert est une des composantes de l'espace public physique.

De manière pragmatique, pour François Leimdorfer, l'espace public est un espace commun à une pluralité d'acteurs, mais dont un pouvoir est garant de l'accès et de l'usage. Il s'agit tout d'abord de l'espace matériel : l'espace de circulation (la voirie : rues, trottoirs, carrefours, places) et les espaces de sécurité (" sous fil à haute tension », canaux d'égouts), les espaces ouverts communs tels que les parcs, les jardins, les marchés, la gare routière et les espaces fermés d’usage public, tels que les écoles, les édifices publics. Il s'agit aussi des espaces moins directement matérialisables, qui impliquent une jouissance de la citadinité et qui mettent en jeu des rapports entre acteurs plus éloignés : la sécurité physique et sanitaire, le bruit, la pollution, le ramassage des déchets, leurs lieux et leurs accès, la circulation des transports. Les espaces privés qui mettent en œuvre un usage public sont à rattacher à cet ensemble, notamment l'utilisation d'un espace privé à des fins publiques (marché, terrasse, piscine, salle des jeux, etc.) (Leimdorfer 1999:53) A ce sujet, les espaces publics comme privés accomplissent le même rôle : l'équilibre biologique (Simon 1952:47).

L'on peut dégager les caractéristiques suivantes dans les aspects politiques et les connotations de ce qui est public : l'espace public se rapporte à l'État et il est communal; il est accessible à tous; il concerne tous; il est un bien commun, voire d'un intérêt commun ou d'intérêt général 
(Fraser 1999:109-142) L'on ajoutera que lorsqu'il est question de public, il s'agit aussi toujours de certaines règles, réglementations et mécanismes de contrôle, qui sont d'application dans l'espace public.

Ainsi défini, l'espace public urbain semble ne rien poser comme problème, car les frontières, de ce point de vue, sont nettes. Si l'on se réfère à son étymologie, l'espace est une réalité ouverte. Cependant, avec l'emprise progressive de la raison scientifique, le concept d'espace s'est conformé peu à peu aux fonctions et aux visées diverses. L'espace devient alors simultanément espace économique, social, résidentiel, culturel. Dès lors, les populations se construisent, au regard de leur passé historique et relativement à la spécificité de leur intégration à l'espace urbain, de leurs propres représentations rationnelles de l'espace. Du coup, les représentations de l'espace deviennent infiniment variées. Toutefois, un consensus s'érige autour de la considération de l'espace comme milieu vital et condition générale de vie, comme appropriation d'un champ d'action et comme contexte de vie sociale.

La représentation de l'espace apparaît ainsi comme un constituant essentiel de la culture, de l'ordre des valeurs et de la mentalité des groupes historiques. Dans la pensée chinoise, le couple espace-temps constitue pour les Chinois un ensemble intimement lié. Il ne s'agit ni de deux concepts abstraits, ni de cadres historiques, mais d'un milieu global spatiotemporel, à la fois milieu réceptif et milieu d'action (Birou 1997:21-30). Cette double réalité est concrètement qualifiée : elle reçoit des qualités selon la manière dont les hommes ensemble l'occupent et s'y meuvent. Ceux-ci peuvent agir directement sur l'espace et le temps par des emblèmes et des symboles qui communiquent non des idées ou des pouvoirs de possession, mais une vertu contagieuse.

Cette conception peut dans une moindre mesure, poser les repères de compréhension des logiques d'actions qui guident certains acteurs à s'approprier des portions d'espaces publics pour leur usage personnel, afin d'y établir des commerces ou d'y percevoir des droits d'usages, car les représentations des espaces sont variées et culturellement déterminantes. C'est au choc de l'organisation de la société, dans une perspective modernisatrice, que la structuration fonctionnelle de l'espace public, jadis considéré comme réalité ouverte, s'érige. Pour comprendre le processus de formalisation de la représentation normative de l'espace public, il importe de saisir comment, d'un espace de vie totale, on est passé aux espaces abstraits.

Compte tenu de cette littérature, nous disons que l'espace public représente d'abord un espace physique : un lieu de rassemblement ou de passage, à l'usage de tous, l'espace de vie collective. C'est un lieu qui n'appartient à personne (en droit), un lieu anonyme, collectif, commun, partagé et mutuel. 
L'espace public représente dans les sociétés humaines, en particulier urbaines, l'ensemble des espaces de passage et de rassemblement ouverts et anonymes, qui sont à l'usage de tous.

Simultanément, c'est un champ de libertés beaucoup plus large que la liberté de circulation, la liberté de manifestation, de parole et d'expression. Il représente aussi un espace moral et symbolique de liberté ne pouvant exister que dans une démocratie dans laquelle les différents acteurs sociaux, politiques, économiques, religieux, culturels, intellectuels peuvent discuter, s'opposer, délibérer. C'est pourquoi il forme le théâtre de l'opposition au pouvoir dans la plupart des conflits politiques et sociaux. Ce champ est cependant restreint par l'ensemble des lois, règlements et pratiques de maintien de l'ordre.

\section{D'un espace de vie totale aux espaces abstraits}

De toute évidence, tout espace humain est un espace de vie. Géographiquement, un espace est la localisation des phénomènes humains visibles. Cependant, la connexion de ces phénomènes humains entre eux, en fonction des ensembles plus ou moins isolés ou complémentaires qu'ils constituent, renvoie à certaines relations abstraites constituant l'existence socio-spatiale des groupes humains. D'autres notions telles que le milieu physiologique et le milieu socioculturel interviennent au-delà de la géographie pour expliquer cette réalité des espaces.

Par rapport à ce qui précède, les notions de milieu physiologique et de milieu socioculturel, fait remarquer Alain Birou, ont l'avantage de faire percevoir une appropriation et une occupation de l'espace, spécifiques de la vie. C'est l'ensemble des constituants qui entourent l'être vivant, dans lesquels il trouve les conditions propres à se développer, à entretenir et à manifester la vie qui l'anime (Birou op.cit.:25). Ce qui permet de distinguer deux ordres de milieu physiologique :

- les milieux cosmiques ou extérieurs entourant l'individu ;

- les milieux organiques ou intérieurs, en contact immédiat avec les éléments anatomiques qui composent l'être vivant.

Au regard de ce qui précède, il apparaît que l'espace humain de vie totale a éclaté. L’homme est soumis aux réalités artificielles qui le modèlent. De même, son espace global (nature et culture) a été submergé par des espaces sectoriels d'un homme devenu tout « raison et calcul ». La conséquence de cette mutation est que l'espace directement économique d'utilisation des ressources, de production et de circulation des biens et des richesses s'autonomise par rapport à l'espace humain total et lui impose ses impératifs. Depuis lors, il se cristallise dans l'espace des complexes institutionnels 
auxquels les hommes doivent se soumettre. Toutefois, la caractéristique la plus importante est, sans doute, non pas l'artificialité de l'espace urbain, mais son envahissement par la mécanisation et la fonctionnalité publique. C'est ce développement fonctionnel de l'espace qui traduit les jeux d'acteurs en termes de conflits inhérents aux différentes logiques d'occupation de l'espace, d'une part, et, d'autre part, à la production des déchets, du fait de cette exploitation de l'espace.

\section{Vers un espace public symbolique}

L'espace public s'institue en espace symbolique ; il ne s'agit pas seulement d'un lieu de rencontre et d'expérience réelle de la citoyenneté, mais aussi d'un espace symbolique, siège de représentations et d'interprétations, objet et enjeux de discours et de désirs. La dimension sociale, politique, économique, environnementale, etc., de la sociabilité consiste justement en la reconnaissance d'une signification et d'une consistance symbolique à l'espace public.

Notons que l'espace public n'est pas un lieu homogène, qu'il ne se limite pas à la place publique, mais qu'il s'éclate en lieux divers dans lesquels ont lieu les activités, elles-mêmes diverses, constitutives de la citoyenneté et de la sociabilité. Des lieux de vie économique (marchés,...) aux lieux culturels (lieux de spectacles, de représentation, les musées, ...), aux lieux politiques (lieux de débats publics, siège des institutions, ...), aux lieux environnementaux (jardin botanique, ...), les lieux de l'espace public déclinent dans l'espace toutes les activités par lesquelles nous donnons à notre société, la réalité effective d'actes et de pratiques. L'espace public est donc plutôt une dénomination générique, constitutive d'une dimension symbolique de ces lieux.

A cet effet, ce qui caractérise l'espace public, c'est, sans doute, que son avènement est lié à l'urbanisation de la cité et de la vie urbaine dans l'espace. C'est l'espace urbain qui comporte des lieux échappant aux usages privés et singuliers pour relever de la loi publique : la rue et la place, espaces publics inscrits dans l'espace, sont des lieux définis dans les espaces urbains.

\section{Espace public et environnement}

Pour qu'il puisse être qualifié d'espace public, un espace doit alors pouvoir être approprié par tous, ce qui sous-entend un certain équilibre de ses fonctions et de ses usages vis-à-vis des citoyens. Il doit en outre être ainsi vécu comme un espace approprié sans possesseur (Arantxa 2000). Dès lors que la propriété est associée à la qualité de vie, au respect de soi, à la bienséance et au bien-être commun, ranger et arranger son espace de vie témoignent d'une volonté de reproduire à l'identique le rapport social que 
l'on entretient avec ceux qui partagent cet espace. C'est ainsi qu'un espace propre est donc celui que l'homme s'approprie en posant des limites et des interdits qui en écartent la saleté. Ces limites et ces interdits sont adressés aux autres avec lesquels l'homme concerné entre en interaction dans un espace partagé. Au-delà de l'espace limitrophe, c'est l'espace public urbain, un espace et des lieux qui sont perçus comme n’appartenant à personne, comme n'étant pas régulé, un peu comme la brousse au village. Il n’est donc pas paradoxal que personne ne s'occupe de la salubrité des espaces publics : ce sont des espaces collectifs et donc sous contrôle politique.

Il faut cependant reconnaître que pour la majorité des populations dans les pays en développement, l'assainissement n'est pas perçu comme un problème qui concerne la parcelle individuelle, mais bien comme un problème collectif qui doit se poser dans le quartier. Or la dimension collective et donc la politique d'assainissement commencent avec l'énonciation et la sanction de normes partagées qui, au-delà des frontières de la cour privée, règlent l'expulsion des déchets hors de l'espace partagé commun. En effet, l'espace privé (la cour d'habitation) est le premier lieu de vie sociale et de production des déchets. Cet espace est en relation avec l'espace limitrophe de la rue qui suit une logique de marquage de l'espace par des dépôts des déchets, puisque la rue, aux abords de concessions, semble être considérée comme un espace vacant, susceptible d'accueillir les déchets de l'espace privé.

La propreté de l'espace public urbain est un signe de l'effectivité du pouvoir politique qui s'exerce sur ce même espace. Inversement, la saleté de l'espace public urbain témoigne de la défaillance des pouvoirs publics locaux et communaux à gérer l'environnement du territoire. Par conséquent, la gestion de l'espace public invite avec insistance le pouvoir, mais aussi la participation des masses populaires et des associations qui peuvent bien prendre en charge collectivement des problèmes de la vie urbaine et participer à la conscientisation populaire autour des thèmes centrés sur des représentations du «bien commun ».

\section{Importance des espaces verts}

\section{Pour le bien-être des humains}

R. Kaplan et S. Kaplan (1989)² ont élaboré une théorie sur le rapport entre l'attention de l'homme et son cadre de vie. Cela signifie que la vie en milieu urbain, avec ses véhicules rapides, ses enseignes au néon clignotantes et ses couleurs fortes, cause un stress permanent. La recherche indique que la végétation et la nature renforcent notre attention spontanée, permettent à notre système sensoriel de se détendre et nous insufflent une énergie nouvelle. Des visites dans les espaces verts sont synonymes de détente et aiguisent 
notre concentration, car nous n'avons besoin que d'utiliser notre attention spontanée. En même temps, nous recevons de l'air frais et la lumière du soleil, qui sont importants pour nos rythmes diurne et annuel.

Par ailleurs, la recherche d'Ulrich et autres ${ }^{3}$ a montré que les patients dans les hôpitaux qui occupent des chambres avec vue sur des arbres guérissent plus vite.

Ces auteurs ont projeté à 120 personnes un film qui fige le sang sur des accidents du travail. Ils ont ensuite passé à la moitié de ces personnes un film sur la nature, alors qu'à l'autre moitié ils ont montré un film sur la ville, avec des séquences montrant des édifices et la circulation. Les pulsations, le tonus musculaire et la tension artérielle des sujets ont été mesurés durant toute l'expérience. Tous les sujets ont manifesté de grands signes de stress pendant le premier film sur les accidents de travail.

Les niveaux de stress de la moitié des sujets qui ont vu ensuite le film sur la nature étaient retournés à un niveau normal après 4 à 6 minutes, tandis que l'autre moitié qui avait vu le film sur les édifices et la circulation a continué d'afficher des niveaux de stress élevés. En Suède, des études approfondies, ont été conduites par P. Grahn sur l'importance des parcs pour différents groupes de population. Elles montrent que les moments passés au grand air ont une fonction curative réelle pour les patients et les résidents des hôpitaux, des hospices de vieillards et des maisons de repos. Les individus étaient plus heureux, dormaient mieux, avaient besoin de moins de médicaments, étaient moins agités et beaucoup plus loquaces. D’autre part, malheureusement, il était évident que les malades, les personnes âgées et les invalides passaient peu de temps dehors. Cela était dû uniquement au fait qu'ils avaient besoin d'une assistance pour sortir. La présence d'arbres et de plantes dans les villes calme et apaise les citoyens, diminue l'insécurité, rend l'environnement plus « humain ».

Une autre étude renchérit qu'à l'intérieur des bâtiments, l'absentéisme et le stress des collaborateurs sont moindres dans les bureaux et les locaux administratifs bénéficiant d'aménagements paysagers (Fare et Claterrbuck 1998).

La dernière étude sur « l'espace vert dans la ville de Bandundu : bilan et perspectives d'avenirs » rejoint celle citée plus haut. Plus l'espace vert est riche en espèces végétales et complexes, plus il donne l'impression de « se ressourcer ». Les rideaux de végétaux réduisent considérablement les nuisances sonores, où le bruit est facilement réfléchi par les surfaces dures. Les plantes absorbent les sons. Les auteurs concluent que dans les hôpitaux, les jardins bien agencés contribuent à réduire le stress des patients et améliorent sensiblement les résultats cliniques (Minte et Malon 2000). 


\section{Pour la biodiversité et l'environnement}

Lors de la Conférence des Nations Unies sur l'environnement et le développement tenue à Rio de Janeiro en 1992, tous les pays participants ont adopté le programme Action 21, plan d'action indiquant comment le monde pourrait parvenir au développement durable. Ce plan les oblige également à œuvrer en faveur du développement durable, obligation qui retombe elle-même sur les administrateurs des espaces verts urbains.

Les jardins et les parcs anciens, sans parler des cimetières, sont souvent visiblement riches en biodiversité. Il s'agit des principaux habitats des végétaux et des animaux. Les installations plus anciennes, bien établies, attirent, par exemple, oiseaux et mammifères dont l'habitat naturel est la forêt.

Etant donné qu'une partie de plus en plus importante de la population vit en zones urbaines et y perçoit chaque jour la nature, celle-ci en milieu urbain est importante pour la sensibilisation à l'environnement et la compréhension de la nature.

La nature telle que l'homme l'a créée est souvent considérée comme inférieure à celle qui se développe sans l'intervention humaine. A l'appui de cette thèse, on affirme souvent par exemple que le nombre d'espèces est souvent plus important dans la nature intacte.

Une chercheuse anglaise, qui possède un jardin d'environ $700 \mathrm{~m}^{2}$ dans la banlieue de Leicester, dans les Midlands (aux USA), est d'avis que ceci n'est pas toujours le cas. Pendant 15 ans, elle a collecté et déterminé les espèces de tous les insectes qu'elle a trouvées dans son jardin. Elle a par exemple reçu la visite de 34 pour cent de toutes les espèces indigènes de papillons, de 30 pour cent de toutes les espèces indigènes de mites et de 36 pour cent de toutes les espèces indigènes de syrphes (Owen 1992:118). ${ }^{4}$

\section{Aperçu sur l'apport historique du développement et la protection des espaces verts}

\section{L'apport des siècles passés}

Tout art ou toute science se doit de ne pas ignorer les expériences faites au cours du passé.

Auparavant, les villes n'avaient ni les proportions ni les densités gigantesques que nous leur reconnaissons aujourd'hui, si bien que les atteintes à la nature ou à l'homme étaient à peu près nulles ou du moins négligeables. Le problème ou les atteintes aux espaces verts se posaient de manière totalement différente de ce que nous avons aujourd'hui, au XXIe siècle, car les énormes concentrations urbaines et la révolution industrielle avec leur cortège des dégradations du milieu naturel, n’ont débuté qu'au XXe siècle. 
A cet effet, quatre grands apports fondamentaux des siècles précédents dans le domaine du développement et de la protection des espaces verts sont à retenir (Soulier 1968).

\section{L'apport plastico-philosophique de l'histoire}

Cet apport a développé davantage la notion de l'art dans les espaces verts. Il a également montré l'importance du développement et de la protection des espaces verts dans les milieux urbains. Le Congrès International d'Architecture Moderne (CIAM), dans La Charte d'Athènes en matière d'environnement (CIAM 1957:18) souligne que cet apport était caractérisé par la notion du beau et de l'esthétique, à tel point qu'à chaque type de jardins correspondait un art particulier.

Précisons que chaque période de l'histoire (Antiquité, Moyen-âge, Renaissance, etc.) a eu sa conception vis-à-vis des espaces verts du point de vue de leur décor, de leur utilisation, de leur développement et de leur protection.

\section{L'apport des recherches physiologiques}

Le plus grand mérite de cet apport est d'avoir montré l'importance ou les fonctions des espaces verts face à l'évolution de la science et des progrès technologiques qui ont caractérisé le XXe siècle.

Les espaces verts contribuent aux conditions de confort normal biologique pour l'homme, aux améliorations spécifiques ou microclimat urbain contemporain, contaminé par les effets de la science et des progrès technologiques (Georges 1961:87).

\section{L'apport de la psychologie}

La végétation dans cet apport exerce une influence considérable, notamment sur le plan psychique. A cet effet, «l'influence des couleurs agit sur l'âme, elles peuvent y exciter des sensations, y éveiller des émotions, des idées qui nous reposent ou nous agitent et provoquent la tristesse ou la gaieté » (Maurel et Brunais 1965:12).

Dans cette influence de l'esthétique, à part la beauté du cadre de verdure que les parcs possèdent en eux-mêmes, l'esthétique des divers paysages urbains renforce des relations harmonieuses entre l'homme et la nature.

\section{L'apport de la sociologie}

La végétation procure des effets bénéfiques sur le plan sociologique pour des raisons diverses, notamment les besoins d'exigences collectives. Depuis plusieurs années, les lieux de rencontres de manifestations nécessaires à la vie communautaire des quartiers ou de la ville ont en partie comme cadre social : les espaces verts ou espaces libres (Rfeitffer 1966:14). 
Aujourd'hui, le besoin des espaces publics et sociaux se fait plus urgent du fait de l'usurpation de cet ancien rôle de la rue par les véhicules; ce besoin va trouver dans les espaces verts un moyen de se réaliser d'une manière adéquate : lieux de détente pour les âges, etc.

\section{Quid de la ville de Kinshasa et de l'espace vert ?}

Capitale de la République Démocratique du Congo (ex-Zaïre), Kinshasa est devenue la plus grande ville d'Afrique tropicale, avec une population qui était estimée en 1994 à 4655000 habitants. Le poste fondé sur la rive sud du Pool Malebo (ancien Stanley Pool) par Stanley en 1881, longtemps connu sous le nom de Léopoldville, devint en 1920 la capitale du Congo belge. A sa création, la ville de Kinshasa comptait 10987 habitants. En 1940, elle comptait 50000 habitants et déjà près de 400000 en 1960, année de l'indépendance.

Depuis cette date, sa croissance s'est accélérée en raison de l'exode rural. Les dernières estimations, selon le recensement administratif de 2005, font état de 7500000 habitants (Le Potentiel 2005). Cette poussée démographique exagérée vers Kinshasa de l'ordre de 30 pour cent de 19992008 (Musinbondo 2008:142) est le résultat de l'exode forcé (dû au déclenchement des épisodes des guerres d'occupation par le Rwanda, le Burundi et l’Uganda (1996-1998 et 1998-2002). Les conséquences de ces guerres ont occasionné les déplacements internes vers la ville de Kinshasa, elles ont posé de sérieux problèmes sur tous les plans. Par exemple sur le plan urbanistique, plusieurs sites ont été envahis (l'immeuble de l'Institut d'Etude Médicale, etc.) et croupissent dans un état de saleté. Il a été aussi noté la construction de taudis dans les espaces verts (exemple : l'espace vert de la $1^{\text {ère }}$ rue Limite, le terrain du camp Kabila, le long de la route des poids lourds, le terminal de la gare de l'ONATRA, etc.) Cette population, estime-t-on, pourrait atteindre plus de 10 millions d'habitants en 2015 et pourrait se hisser ainsi parmi les 30 plus grosses agglomérations mondiales (United Nations 2007).

De son site original (Galiema), la ville s'est étendue vers l'amont (Ndolo), vers l'aval (Kalina), puis vers le sud, dans une zone coupée de dépressions marécageuses. Le contraste est grand entre les types de quartiers. Le centre des affaires, la zone administrative, plusieurs zones résidentielles se trouvent au bord du fleuve. Un urbanisme moderne a dégagé de larges avenues, multiplié les grands buildings, mais aussi les espaces verts, disséminé pavillons individuels et villas luxueuses entourées de gazon, d'arbres et de fleurs. C'est le cœur même de la ville, situé en arrière du port qui, bien équipé, est le point de jonction entre les lignes fluviales et la voie ferrée de Matadi, achevée dès 1897. 
A l'époque coloniale (1950-1960), la physionomie de la végétation urbaine de Kinshasa présentait une répartition hétérogène des types d'arbres selon les quartiers. Aussi distingue-t-on deux ensembles : l'un qui est bien boisé, avec une concentration de jardins et des artères bordées d'arbres en plantations monospécifiques ; l'autre, densément bâti, qui offre peu d'artères, de jardins et de cours arborés. Cette différence s'explique à la fois par des faits historiques et par l'organisation actuelle du milieu urbain entre le centre et la périphérie. Le centre administratif, les quartiers résidentiels ont bénéficié des plantations d'arbres et de l'entretien pour lutter contre les vents et l'érosion, pour leur ombrage et l'embellissement de la ville, excepté les manguiers et cocotiers. Par exemple : au boulevard Lumumba long de 17 $\mathrm{km}$, les arbres (l'espèce peltophorum plerocarpun et les eucalyptus ) étaient plantés et alignés pour orner le long des artères d'accès de part et d'autre de la chaussée formant une sorte de haie ; l'espace de la radio et télévision congolaise (100 hectares) et d'autres cours des bureaux, les arbres ornés et fleuris (l'espèce cananga odorata) étaient aménagés et plantés aux cours qui occupaient l'espace vide, servant de l'ombrage ; l'espace vert ONPTZ à Masina et du Camp Kokolo (200 et 150 hectares), les arbres (l'espèce eucalyptus et tectona grandis) de plantations intra-urbaines constituaient les peuplements artificiels, généralement mono-spécifiques, d'essences exotiques. Avec, comme spécificité, ornements et ombrage, etc.

A cette époque, les acteurs principaux qui intervenaient dans la gestion des espaces verts étaient le Ministère des affaires foncières et celui de l'environnement et de la conservation de la nature, qui prenaient des décisions au Congo-belge, sous la bénédiction du Roi Léopold II de Belgique.

Il faut aussi souligner que les espaces verts de cette époque suivaient la logique du Centre-périphérie (une logique qui a donné naissance à la discrimination raciale et toutes ses conséquences sur le plan social). Les " espaces verts » de la périphérie où vivaient les Noirs couvraient la forêt naturelle. Son aménagement et sa croissance dépendaient plus de la nature. En revanche, au centre où l'homme blanc résidait, les espaces verts couvraient les lieux aménagés pour les jeux, la récréation, les jardins, les promenades, les ornements des routes et carrefours, etc. L'administration coloniale, à travers le service des espaces verts de la mairie, s'occupait de son entretien.

Après l'indépendance, vers les années 60 , de profonds bouleversements qu'à connus la RDC, amenèrent le chef de l'État, le Président Mobutu, à prononcer un discours, précisant que «le sol de nos ancêtres ne pouvait plus faire l'objet d'une quelconque appropriation ». Ce discours avait modifié l'ordonnance de la gestion des terres et a donné naissance aux textes juridiques ci-après : 
- Loi Bakajika nº66/343 du 7/06/1966. Cette loi rendait seulement les terres et les concessions appartenant aux particuliers et aux sociétés privées à l’État.

- La loi constitutionnelle du 31/12/1971 qui précise que le sol et le sous-sol deviennent la propriété exclusive, inaliénable et imprescriptible de l’État congolais, cette loi renforça la loi Bakajika.

Juridiquement et administrativement, la gestion des espaces verts reconnaît six acteurs (intervenants) dans la gestion des espaces verts en RDC en général et à la ville province de Kinshasa en raison de leur participation, à savoir :

1. le Ministère des affaires foncières ;

2. le Ministère des travaux publics et infrastructures ;

3. le Ministère de l'urbanisme et de l'habitat ;

4. le Ministère de l'environnement et de la conservation de la nature ;

5. les bailleurs de fonds ;

6. la population.

Pour ce qui est de la gestion des espaces verts, deux services spécialisés rattachés au Ministère de l'environnement et de la conservation de la nature s'occupent de la protection des espaces verts dans la ville de Kinshasa : il s'agit du Service de ceinture verte et le Service national de reboisement.

\section{Service de ceinture verte}

Il s'occupe de l'aménagement horticole (les pelouses et gazons constituant les arbres à haute tige). Il s'occupe aussi de l'entretien des parcs d'attraction et d'agrément, des jardins botaniques et zoologiques, des ronds-points, de la pépinière, de la construction et de l'entretien des bandes vertes pour les piétons et de l'ornement de la ville pendant les fêtes.

\section{Service national de reboisement}

Ce service s'occupe de trois sortes de reboisements ci-après :

- le reboisement de reproduction (il plante des arbres pour la production de bois de chauffage, de cuisson, de construction) ;

- le reboisement de protection (qui lutte contre les érosions, les gaz carboniques et à effet de serre, atténue les bruits des moteurs) ;

- le reboisement d'agrément (en vue de créer des espaces verts qui servent à des loisirs, à la détente, aux promenades, etc.). 
Il convient de relever que tous ces services restent de nos jours des canards boiteux ; on les trouve seulement présents au palais de la Nation (bureau du Président de la République), au Palais du peuple (au Parlement et au Sénat), on les voit également travailler en synergie avec quelques sociétés de la place dans le cadre de la publicité de leurs produits (exemple : rond-point FORESCOM aménagé par Vodacom (une entreprise téléphonique), Rondpoint Lemba super par Shell, terrain Golf par la Bralima, etc.).

\section{État des lieux des espaces verts dans la ville de Kinshasa}

Le plan régional d'urbanisme de 1967 avait divisé la ville de Kinshasa en deux parties, compte tenu de la dualité « urbanisé » et «non urbanisé ». L'ouest de la ville de Kinshasa regroupe les communes suivantes : Ngaliema, Kitambo, Gombe, Bandalungwa, Ngiri-ngiri, Kasa-vubu, Lingwala, Kinshasa, Barumbu, Selembao, Bumbu et Mont Ngafula. A l'est, il y a les communes ci-après : Kalamu, Makala, Ngaba, Lemba, Limete, Kisenso, Kimbaseke, Ndjili, Masina, N'sele et Maluku.

La capitale Kinshasa présentait un caractère discriminatoire. Les communes ouest habitées par les Blancs (Gombe, Kitambo et Ngaliema) comportaient de nombreuses infrastructures et des équipements collectifs. La partie ouest reflétait l'image de l'Europe. Et sur le plan esthétique et urbanistique elle répondait aux normes urbaines. En revanche, la partie est de la capitale, la moins urbanisée, comportait la construction des cités planifiées à l'image d'une ville ancienne. Mais c'est à partir de la décennie 70 que la gestion des espaces verts a commencé à projeter une figure de deuil.

Aujourd'hui, la ville de Kinshasa offre l'image d'une énorme poubelle. De «Kin-la belle » (ou Kinshasa la belle), qualificatif de l'aspect resplendissant de la capitale de la RDC dans les décennies 60-70, on a viré dans l'autre extrême pour désigner actuellement cette ville par ce que les médias et la population nomment désormais par "Kin-la poubelle » (ou Kinshasa la poubelle). Devant les désordres imprescriptibles dans la gestion des déchets urbains, les jeunes Kinois ont rebaptisé certains coins de la capitale : «Iran-Irak », " Rive gauche », "Sarajevo » ou, plus récemment, « Kosovo », " Tchétchénie », « Afghanistan », « Bagdad », 5 à cause de l'entassement combien spectaculaire des déchets expliqué, entre autres, par la quasi-inexistence des services de collecte des déchets urbains, lesquels ne sont plus assurés que dans quelques communes. En 1992, dans le reste de la ville, comme en témoignaient déjà Hardoy et Satterwaite, ${ }^{6}$ les sacs plastiques jonchaient les allées, les déchets étaient déposés sur la route, soit déversés dans les égouts ou enfouis dans des décharges improvisées et à ciel ouvert. Ils sont parfois incinérés, mais sans précaution aucune ni sur l'état de l'environnement, encore moins sur celui de la santé publique. Du 
matin au soir, les citadins de bon nombre de quartiers de Kinshasa vivent avec et dans la saleté. Ces rejets dans les décharges improvisées ainsi que d'autres pratiques de collectes des déchets urbains ont des conséquences sévères sur les espaces verts de la ville de Kinshasa.

Pour besoin d'analyse, cette section rend compte de l'état de lieux des espaces verts à Kinshasa dans la partie ouest, d'une part, et est, d'autre part.

\section{Les espaces verts dans les communes ouest}

Nous avons retenu au total neuf espaces verts, à savoir :

Le terrain du golf

Cette ceinture verte héritée de l’époque coloniale est située dans la commune de la Gombe. Il longe le boulevard du 30 juin et se situe entre le cimetière de la Gombe et le bâtiment du Ministère du travail et de la prévoyance sociale. Ces immenses espaces verts étalés présentent un paysage d'une beauté exceptionnelle servant aux compétitions de golf (SDAU 1975). C'est le seul espace vert de la ville de Kinshasa qui, bien que spolié, est resté bien entretenu et bien protégé, dans le cadre de la publicité faite par certaines entreprises de la place.

\section{Le jardin zoologique}

C'est également une ceinture verte composée par la majorité d'arbres (espèces : hura crepitans, milicia excelsa, tectona grandis, trema guineensis, arancaria cunninghamii, acacia spp, ... qui ont l'intérêt particulier de fournir de l'ornement et et de l'ombrage) à haute tige, pour la protection des animaux, notamment contre le soleil, le gaz carbonique et d'autres gaz à effet de serre, les poussières, etc. Cet espace vert est dans un état d'abandon. La couverture végétale est en voie de disparition. Beaucoup d'arbres sont détruits ou abattus. Les animaux meurent de faim et on y observe une carence de diversité d'espèces animales. Sa valeur touristique est réduite à sa plus simple expression. Terrasses et restaurants de fortune occupent les ruelles du jardin.

\section{Jardin botanique (Parc de Boeck)}

Situé dans la commune de la Gombe et comprise entre l'avenue du Commerce et Rwakadingi, cet espace vert et verdoyant de la capitale est dans un état d'abandon total, les différentes espèces d'arbres locaux et importés sont détruits. Il y reste quelques végétaux en termes de plantes conservées dans les pépinières souvent mal entretenues. Le terrain est spolié par la construction de boutiques (BEAU 1985).

Les espaces verts Mont Fleury

En 1970, il était question de construire dans ce site des équipements collectifs de grande envergure. Aujourd'hui, ces espaces sont vendus et envahis par 
des maisons d'habitation d'hommes appartenant à des couches sociales financièrement fortes. Les espaces verts n'existent plus, les gens construisent même sur le site érosif.

Les espaces verts de Mbenseke Futi

Cet espace que l'Hôtel de ville avait réservé pour la construction d'une nouvelle cité est transformé en grande partie en cimetière. D'autres parties sont envahies par des maisons en construction anarchique et, en amont, les gens profanent même les tombeaux par des constructions anarchiques. En aval, ce sont les jardins, avec toutes sortes de conséquences sur la santé humaine.

\section{Les espaces verts de Mitendi}

Cette localité est devenue aujourd'hui une grande cité non urbanisée, et les constructions poussent à une vitesse de croisière. Il n'existe plus d'espaces verts.

Les espaces verts de l'Institut d'Etudes Médicales (en face de camp Kokolo)

Cet immense espace vert où était construit l'Institut d'Etudes Médicales (IEM) est dans état de détérioration très avancée. Il a été laissé par le pouvoir colonial pour une implantation d'équipements collectifs futurs ayant trait au domaine médical. L'immeuble inachevé, derrière l'IEM, devrait être le plus grand hôpital général du pays, malheureusement il est habité par les familles de militaires, de déplacés internes des conflits armés (1996-2002). Présentement, ces espaces verts sont vendus aux confessions religieuses (catholique, kimbanguiste, protestante, Mpeve ya longo) qui les ont transformés les unes en maisons d'habitation, les autres en églises et en écoles, etc. Toutes ces constructions sont érigées sous le label du service cadastral et sous l'œil impuissant de l'Hôtel de ville.

Les espaces verts de Mbinza Météo

Ils couvrent les infrastructures du Centre National de Météorologie de la RDC. Le centre a été construit au milieu d'une grande étendue d'espaces verts pour émettre les ondes sans faille. Le terrain a été envahi il y a à peine un an par des constructions anarchiques, en complicité avec le comité de gestion de cette entreprise publique et le service cadastral.

\section{La forêt de Lukunga}

Cette forêt a été bornée en 1957 pour le compte de la société d’État REGIDESO. Cet espace vert avait pour but de protéger la cour d'eau de Lukunga, l'usine de traitement d'eau qui alimente une partie de la ville de Kinshasa et de construire des maisons modernes pour la société. 
Les paysans ont exterminé les arbres pour la fabrication de la braise. De nouvelles cités ont été construites, notamment : la cité Maman Mobutu (urbanisée), et la cité Matadi-Kibala (non urbanisée).

\section{Les espaces verts dans les communes Est de la ville de Kinshasa}

Nous avons dénombré dans cette partie de la capitale huit espaces verts, notamment :

\section{Espaces verts ONPTZ (Masina Sans-fil)}

Espace abritant les installations téléphoniques de l'ONPTZ redevenu OCPT. Les 1000 hectares d'espaces verts de cette entreprise publique ont été lotis à la hâte et sans respect des normes urbanistiques, en l'absence totale d'égouts et de caniveaux se distinguent par des avenues bloquées, à tel point que quand il pleut, la nouvelle cité se transforme en lac. Il faut attendre plusieurs heures pour que les eaux de pluie se filtrent. Même les murs du bâtiment de l'OCPT partagent mitoyennement plusieurs parcelles.

\section{Espaces verts le long du Boulevard Lumumba}

De part et d'autre, les arbres (espèces : peltophorum, plerocarpun et les eucalyptus) ont été plantés pour ornement, afin de diminuer les bruits des véhicules et les gaz qui ont des effets néfastes pour les passants et les maisons environnantes. Certains arbres ont vieilli, d'autres sont coupés sans raison par la population.

Bon nombre d'espaces sont achetés par les pétroliers. On compte aujourd'hui dans ces espaces 12 stations de carburant. Par ailleurs, le long du boulevard est envahi par les maraîchers, et plusieurs décharges improvisées $\left(1^{\mathrm{e}}, 2^{\mathrm{e}}\right.$ et $15^{\mathrm{e}}$ rue Limete) et des déchets industriels perdent l'esthétique d'un boulevard (SDAU 1975). L'absence d'entretien des arbres et des trottoirs doit aussi être mise en relief.

\section{Espaces verts eucalyptus (N'djili quartier 1)}

Pour plus de précisions, disons que la commune de N'djili compte trois espaces plantés d'eucalyptus. L'un au boulevard Lumumba qui a connu un certain reboisement mais plusieurs arbres ont été détruits en faveur d'une station à carburant (compagnie française ELF). Récemment, le reste d'espace a été transformé en marché de récupération des déchets et plusieurs terrasses ont été érigées. Mais le gouverneur de la ville n’a pas tardé à les démolir. Celui de l'intérieur (à côté du marché Mangobo) a été vendu aux grossistes qui ont construit des dépôts. L'autre espace (à côté de l'auditorium militaire) a été vendu aux particuliers par le bourgmestre de la commune de N'djili. 


\section{Espaces verts de Kinkole}

Cet espace a été affecté par le pouvoir colonial à la construction de cités satellites. En 1975, dans son discours "retroussons les manches », ${ }^{7}$ le président Mobutu déclara que cet espace avait comme objectif la création de fermes agropastorales et de champs à caractère économique. Cet espace s'est vu morceler par les dignitaires du parti unique, le Mouvement Populaire de la Révolution (MPR). Seul le président Mobutu a réussi à construire une usine alimentaire (DAIPN). Cette étendue est de nos jours envahie par des lotissements faits de façon anarchique. Et une cité improvisée a été créée à la hâte pour les familles des déplacés internes des guerres, à l'est du pays.

Espace vert CREN-K (Centre de Recherche et d'Etudes Nucléaires de Kinshasa) dans le site de l'Université de Kinshasa.

Plusieurs têtes d'érosions menacent cet espace vert (Cren-K) qui garde l'uranium et d'autres métaux nocifs pour la recherche scientifique. L’Université de Kinshasa et le Ministère de la recherche scientifique gèrent impuissamment cet espace.

\section{La forêt galerie de Limete résidentiel ( $7^{\mathrm{e}}$ rue Limete)}

Cette forêt n'existe plus et il n’y a aucune trace de végétaux. Elle était réputée pour les avocatiers plantés le long de la route. Les Kinois l'avaient dénommée «Zamba ya avocat » (Champ d'avocat). Cet espace est envahi aujourd'hui par des constructions de gens ayant des moyens financiers.

\section{Vallée de Lukaya}

Gérée par l'église catholique (archidiocèse de Kinshasa), la grande forêt, qui couvrait cet espace vert, est en train d'être décimée par la production de braise et de bois de chauffe.

\section{Causes déterminantes de la destruction et de la spoliation des espaces verts à Kinshasa}

Pour mieux déceler ces causes, nous distinguons d'une part les causes juridiques et conjoncturelles, et, d'autre part, les causes structurelles.

\section{Les causes d'ordre juridique}

En regardant de près l'état dans lequel se trouve la ville de Kinshasa, capitale de la République Démocratique du Congo et siège des institutions, il y a lieu de se poser la question de savoir s'il existe des lois qui accompagnent la politique publique dans la gestion des espaces verts.

Dans « la morale de L'État à la morale de l'Administrateur », on fait observer ceci : 
Il ne saurait donc exister de divorce prolongé entre le comportement de l'État et celui des citoyens qui le composent. Et si l'État est apparemment amoral, voire immoral dans son action, c'est-à-dire dans ses lois, dans les procédures et dans les fins qu'il poursuit, c'est que les hommes, tour à tour responsables de ces lois, de ces procédures et de ces fins, y ont projeté leur propre égoïsme, leur appétit de puissance et leurs propres carences(Catherine et Thouillier 1982:9).

Cela étant, au niveau du sommet, depuis l'époque coloniale en passant par l'indépendance jusqu'aujourd'hui (en 2009), il y a des ordonnances, des arrêtés et des décisions qui ont été pris portant application des mesures de la politique publique de la protection et d'assainissement des espaces verts en RDC en général et dans la ville de Kinshasa en particulier. Mais que sont devenues toutes ces mesures ?

A cet effet, deux tendances s'affrontent par rapport à la protection de la politique publique des espaces verts en RDC.

La première tendance que nous qualifions de minimaliste, estime qu'il y a dans ce pays en général et dans la ville de Kinshasa en particulier une politique nationale de gestion des espaces verts qui se trouve de manière directe et indirecte consignée dans l'ordonnance présidentielle n 75/231 du 22 juillet 1975, fixant les attributions du Ministère de l'Environnement, de la Conservation de la nature et du Tourisme et complétant l'ordonnance $n^{\circ} 69$ 146 du $1^{\text {er }}$ août 1969 qui fixait le nombre de ministères, leurs dénominations et leur compétence respective. Cela prouve que les espaces verts sont gérés par un ministère et des services.

La deuxième tendance est celle maximaliste qui considère, quant à elle, qu'il n'existe pas une politique nationale et urbaine en matière de gestion des espaces verts, car il n’y a pas de volonté politique clairement exprimée. Par rapport à ces deux tendances, nous pensons que s'il existe en RDC des textes juridiques qui régissent la gestion et la protection des espaces publics, il n'y a pas une volonté politique clairement exprimée pour les appliquer. Les faits suivants permettent de légitimer notre thèse.

\section{Sanction de l'inobservance des textes juridiques}

La lecture des textes sur la gestion des espaces verts révèle trois catégories de sanctions :-administrative (elle consiste essentiellement en un « retrait du permis d'exploitation » aux privés gérant les espaces verts sans respect de l'hygiène et de l'assainissement, ${ }^{9}$ exemple : jardin botanique, jardin zoologique, eucalyptus de N'djili quartier1, etc.) ; - pénale (la servitude pénale (la peine de prison) inférieure à 5 ans et l'amende) ; ${ }^{10}$ - civile (elle consiste en une réparation en nature ou en numéraire) ». ${ }^{11}$ Malheureusement, la plupart de ces sanctions ne sont pas appliquées et les acteurs impliqués 
dans la destruction des espaces publics, même des espaces verts, restent impunis.

Lacunes des textes juridiques

La lecture de la législation montre qu'un seul texte juridique réglemente souvent plusieurs aspects : toutes sortes d'abattage d'arbres (dans l'espace vert naturel et artificiel), leur gestion (BEAU 1990:25.), etc., la conséquence directe est que souvent ces textes sont superficiels. La sanction doit donc être limitée dans sa description qualitative et non quantitative.

\section{Anachronisme et obsolescence des règles}

A part quelques-uns d'entre eux, presque tous les textes qui gèrent les espaces verts datent de l'époque coloniale. Il est significatif d'indiquer que les préoccupations environnementales ne rivalisent avec les autres sujets d'actualité qu'au lendemain de la conférence de Stockholm de 1972, et surtout à la suite du sommet de la terre (1992) et du sommet mondial sur l'habitat (1994). Il est impérieux de souligner que les lois de la gestion des espaces verts d'une société moderne doivent s'aligner aux exigences naturelles, compte tenu des progrès technologiques.

Quasi-absence des réglementations communales

Le solde de la réglementation sur l'assainissement, l'hygiène, l'environnement en général, et les espaces verts en particulier, se révèle largement débiteur à l'échelle des communes. La question ne semble pas avoir retenu l'attention des autorités municipales et des quartiers. Kinshasa est loin d'échapper à ce qui est devenu une véritable règle. C'est face à un tel constat que le gouverneur de la ville de Kinshasa a pris l'arrêté n SC/0039/BGV/KIN/2007 du 27 mars 2007, portant application des mesures d'assainissement des espaces verts et de protection de la salubrité publique dans la ville de Kinshasa.

Mais ces mesures ne sont pas souvent accompagnées de suivi et de dispositifs institutionnels (Brigade urbain de l'assainissement, Service de ceinture verte, Service national de reboisement et population). Devant cette impasse, toutes les mesures prises dans ce domaine restent lettre morte.

\section{Les causes d'ordre conjoncturel}

Il s'agit des causes qui sont liées à l'évolution sociale, au problème et aux contingences que traverse la RDC en général, la ville de Kinshasa en particulier. A ce sujet, nous avons retenu les causes ci-après :

\section{La crise de logement}

Les ressources en espaces verts sont l'objet d'une forte pression. Depuis 1970, le pourcentage de la population vivant en zones urbaines augmente rapidement et le manque d'espace pousse les populations à construire sur 
les espaces verts. Même là où il y a des édifices publics, des musées, des terrains des jeux par exemple, où l'espace vert reste accessible au public, dans de nombreux cas, cela diminue la qualité récréative générale de l'espace. Cela est surtout perceptible dans les périphéries de la ville de Kinshasa où la pression se fait le plus sentir, là où la ville planifiée fait place aux établissements créés spontanément. Il en est de même dans les zones où le taux de croissance démographique est le plus élevé et/où les administrations municipales contrôlent le moins l'aménagement des sols.

De 1960 à 1965, deux organismes publics ont réalisé au total 2000 logements. La dernière réalisation de l'Office National de Logement (ONL), de 1975 à 1985, comprenant une centaine de logements, n'a pas touché toute la couche sociale à revenus modestes (Beau 1990:25). La dissolution de l'ONL en 1982 et celle de la Caisse Nationale d'Epargne et de Crédit Immobilier (CNECI). La crise ou l'absence de politiques publiques en matière de logement et de l'habitat a été à la base d'habitations et de constructions anarchiques qui, à leur tour, ont entraîné les dommages environnementaux, notamment la destruction des espaces verts.

De 1974 à 1999, le secteur privé, avec l'apport des ONG et l'appui des églises protestantes américaines, avait construit 2266 logements en RDC, dont 146 à Kinshasa. La continuité de cette œuvre par les Congolais qui visaient à construire encore plus de maisons, s'est soldée par une mauvaise gestion. Mis à part cela, il y a eu également toute une série de crédits immobiliers (crédit Luymas, Marana Line du fils de l'opposant politique Etienne Tshisekedi, etc.) patronnés par les Congolais. Et tous ces crédits immobiliers se sont soldés par une escroquerie.

En 1986, 442 logements à la Cité verte et en 1988, 674 logements à la cité Maman Mobutu ont été construits avec l'intervention du pouvoir public, par le truchement des ressources de deux entreprises publiques (Société Nationale d'Electricité et Régie des Eaux).

Toutes ces initiatives privées et publiques dans la production de logement présentent aujourd'hui un signe d'essoufflement et d'effritement. Certaines sociétés, ONG et crédits immobiliers n'ont existé que de nom. Cela a entraîné une occupation désordonnée des espaces verts urbains et conduit à la destruction des espaces verts et à la création de taudis dans la périphérie de la ville.

\section{L'explosion démographique}

Rappelons que la population congolaise double tous les 25 ans. Elle est passée de 15 millions d'habitants en 1965 à 47 millions en 1995. Le taux annuel de croissance est resté supérieur à trois pour cent au cours des 40 dernières années. Devant la pauvreté accentuée, l'exode rural et forcé a provoqué un afflux de la population vers Kinshasa. Cette croissance démographique non 
maîtrisée et incontrôlée a donc entraîné des répercussions néfastes sur le logement et surtout sur les réserves foncières. L'absence d'une politique démographique apparaît également comme une des causes principales des destructions des espaces verts.

\section{Le phénomène d'urbanisation}

L'urbanisation dans la ville de Kinshasa est vue par plus d'un observateur comme un facteur majeur qui influe négativement sur les espaces verts et libres. Actuellement, dans les divers coins de la ville de Kinshasa, il est impossible de maintenir des anciennes lignes de démarcation qui ont séparé l'homme des espaces verts ou de la nature. Les cas plus illustratifs sont ceux qui concernent les communes suivantes: Selembao, Mont-ngafula, Kimbanseke, Masina, etc. La croissance urbaine dans la ville de Kinshasa s'effectue en flèche et entraîne la disparition des espaces verts.

\section{La déforestation}

La première idée des Kinois quand ils sont en face d'une forêt, c'est la production de bois de chauffe ou de braise, mais aussi l'usage du bois pour la construction des maisons. On note à cet effet que cinquante huit pour cent des ménages utilisent du bois ou de la braise pour préparer le repas (Rapport annuel, Centre d'Adaptation des Techniques Energie-Bois, 2000:8), malgré la présence en RDC du grand barrage d'Afrique (Inga). Ce phénomène majeur contribue aussi à la destruction sensible des espaces verts.

\section{La crise de l'État}

Toutes les causes de la disparition des espaces verts que nous venons d'évoquer sont le reflet de la crise de l'État qui se traduit par le déficit de moyens de régulation sociale ou d'interventionnisme dans les divers secteurs de la vie quotidienne. La crise de la gouvernance environnementale et celle des espaces verts en particulier n'ont pas tellement fait l'objet de beaucoup de réflexions en RDC. La littérature sur la gouvernance dans le domaine des sciences sociales est encore dominée par des études qui mettent en relief la situation de l'État dans ses fonctions économiques, politiques et sociales, parfois dans ses missions ayant partie liée avec la résolution des conflits armés ou la lutte contre la corruption.

Ainsi, une des dimensions pertinentes de la crise de l’État est celle qui concerne la mauvaise gestion des espaces publics territoriaux. Si le territoire est l'une des composantes sociologiques de l'État, si l'hygiène et la santé sont utiles pour le développement du capital humain, si les activités récréatives sont importantes pour la santé mentale de la population, alors la question de l'analyse des espaces publics physiques ne saurait être reléguée à l'arrièreplan. C’est le grand mérite de cette étude : celui d’avoir déplacé le débat 
vers un domaine de recherche peu exploré, de repenser l'État par un autre point d'entrée : les espaces verts.

\section{Les causes d'ordre structurel}

Ces causes s'inscrivent dans la durée et sont liées notamment au dysfonctionnement des cadres institutionnels en matière d'environnement et de conservation de la nature, mais aussi à l'absence d'une éducation environnementale.

Concernant le cadre institutionnel en matière d'environnement et de conservation de la nature, il y a un certain nombre de déficiences qui s'érigent en obstacles pour une protection rationnelle des espaces verts, à savoir :

- la mauvaise gouvernance environnementale ;

- l'absence d'appui aux institutions concernées par la gestion des espaces verts ;

- l'insuffisance des moyens financiers, techniques, matériels et humains pour mieux gérer les espaces verts du point de vue de leur conservation, protection et développement.

L'absence de l'éducation environnementale, le fait que la majorité de la population kinoise n'a pas d'éducation environnementale, sont visibles notamment à travers les comportements négatifs qu'affichent les Kinois vis-à-vis des espaces verts ou encore de l'utilisation des sols.

\section{Les conséquences de la destruction des espaces verts}

\section{Du point de vue écologique et environnemental}

L'émergence et la prolifération des érosions

Les espaces verts constituent de précieux matériaux qui freinent les érosions et facilitent la retenue de la pénétration des eaux.

La ville de Kinshasa enregistre plus de 200 têtes érosives, causant de nombreux dégâts matériaux. Entre 1987 et 1990, le Bureau d'Etudes et d'Aménagement Urbain (BEAU) a dénombré 3875 logements détruits par des catastrophes naturelles. Ce chiffre est passé à 6450 en 2000, contre 3875 en 1990 (BEAU 2000:15) Tous ces dégâts sont les résultats d'une mauvaise gestion des espaces verts par l'autorité urbaine.

La destruction, voire la disparition des écosystèmes Faisant allusion à l'habitat végétal, la rivière N'djili, qui traverse quatre communes de Kinshasa, avec sa verdoyante forêt pour le besoin des cultures maraîchères, a donné place à des taudis et à des quartiers flottants à la saison de pluie et qui ont détruit tous les écosystèmes. 


\section{Du point de vue de la santé publique}

Les espaces verts sont une nécessité vitale qui concourt à une santé saine de la population. Cela étant, les fonctions régénératrices des espaces verts ont pour rôle: l'épuration de l'air, l'amortissement des bruits et la fixation des poussières, l'équilibre hygrométrique de l'atmosphère des masses profondes de végétations diverses, qui sont indispensables : arbres de haute tige, arbustes, gazons, etc. (Vitron 1965:15).

En ce qui concerne le gaz carbonique, les odeurs nauséabondes, la ville de Kinshasa bat le record de l'insalubrité des villes africaines et du monde, ${ }^{12}$ sans compter les décharges improvisées et béantes; le rejet des déchets ménagers dans les ruisseaux dégage excessivement. Cela est dû surtout à l'exode rural non réglementé, qui a entraîné les comportements de la campagne à la ville et l'absence d'une éducation urbaine ayant occasionné la disparition de bon nombre d'espaces verts.

A propos de bruit, depuis plus de deux décennies, la ville de Kinshasa assiste à une montée irrésistible de bruits provenant des terrasses en plein air, des groupes de prière, des vrombissements des véhicules, des tôliers et garagistes travaillant sur les places publiques, des concerts des musiques (profanes et religieuses), des veillées de prière, etc. Tous ces bruits finissent par entraîner les circonvolutions du cerveau. Sur le plan environnemental, la solution peut provenir du maintien des espaces verts ou de la création d'autres espaces verts, ou alors il faudrait planter des arbres qui atténuent les bruits et les sons de haute fréquence. Par ailleurs, on peut évoquer aussi la ventilation du vent qui détruit les logements, les arbres, ainsi que l'accumulation des poussières et la température élevée, du fait de l'absence de grands arbres.

\section{Du point de vue économique}

Précisons que les espaces verts revêtent une valeur économique, car ils sont porteurs de nombreuses richesses qu'on peut capitaliser. "Presque près de trois quarts de la population du globe est en majorité dans les pays en voie de développement et dépendent directement des plantes comme sources de médication » (Falloux et Talbott 1995:51). Kinshasa compte un nombre suffisant d'officines traditionnelles. La pauvreté dans laquelle se trouve la population l'a amenée à se faire traiter par les feuilles, les écorces d'arbres et les racines qui procurent des sommes énormes aux tradi-praticiens.

Il y a aussi des lieux touristiques, comme la vallée de Lukaya. Dans sa verdure, l'espace vert du jardin botanique (parc de Boeck) qui peut générer les intrants, est affecté à l'organisation des funérailles ; celui du jardin zoologique, plusieurs terrasses et restaurants de fortune y sont érigés et cela crée un manque à gagner pour le trésor public. 


\section{La gestion des espaces verts par l'autorité urbaine et la population}

\section{L'autorité urbaine}

L'aménagement de la gestion des espaces verts dans la ville de Kinshasa a débuté avec le plan de 1950 par l'administration du Congo belge. Le plan régional de 1967 est venu réaliser les équipements, les infrastructures nécessaires au bon fonctionnement de la ville. En 1975, le schéma directeur d'aménagement et d'urbanisme est venu prendre en compte l'ensemble des problèmes posés par une croissance démographique. Enfin, le projet de développement urbain de Kinshasa de 1985 avait pour but de développer et d'entretenir les infrastructures urbaines, d'améliorer les services urbains et les espaces publics.

Depuis la période coloniale, tous les projets d'aménagement de la ville de Kinshasa sont restés lettre morte. Les espaces verts, surtout, sont restés dans un état déplorable et chaotique. Tous les locataires de l'Hôtel de ville de Kinshasa qui se sont succédé (depuis Henry Stanley Mortor en 1881 jusqu'en 2009 avec le gouverneur de la ville André Kimbuta Yango) n’ont rien changé à cette situation. Certes, il sied de dire qu'à l'époque coloniale, l'aménagement de l'espace vert était bien entretenu là où vivait l'homme blanc. Après l'indépendance, les espaces laissés par les colons et ceux gérés par les natifs se sont dégradés. La nature semble être le cadet des soucis de l'autorité urbaine. Ainsi, pour marquer son incapacité dans la gestion de la salubrité publique urbaine, l'ex-gouverneur de la ville de Kinhsasa, Bernardin Mungul Diaka (1992-1996), dira à la population : « Bakombolaka ndako ya matanga té » (on ne balaye pas une maison en temps de deuil). Une semaine après, la ville s'est retrouvée avec les caniveaux bouchés, les déchets urbains remblayés çà et là.

\section{La population}

A cause du manque de culture et d'éducation environnementale, les espaces verts de la ville de Kinshasa sont devenus des lieux de dépôt des déchets, des spoliations pour des constructions anarchiques, des marchés pirates ; la nuit, les policiers et les malfaiteurs de tout acabit s'en servent pour leurs forfaits, etc. La population ne connaît donc pas l'importance de l'espace vert.

Quelle relation entre espace vert et espace public ?

Tout au long de cette dissertation, nous nous sommes efforcé d'expliquer le contour du concept «espace public » dans son fondement historique et dans son explication contemporaine. La question est de savoir en quoi l'espace vert est un espace public. L'espace vert, nous l'avons dit, représente d'abord 
un espace physique et verdoyant : un lieu de rassemblement, de retrouvailles ou de passage, à l'usage de tous, un espace de vie collective. Ensuite, c'est un lieu qui n’appartient à personne. Un lieu anonyme, collectif, commun, partagé, mutuel et à l'écoute de la nature. Il peut être public ou privé, payant ou non, selon le cas. Ces attributs de l'espace vert prouvent à suffisance qu'il n’y a pas une séparation avec l'espace public. Donc, l'espace vert est un espace public.

Cette affirmation est visible dans ces deux sites : au jardin botanique de Kinshasa (JBK) et au jardin zoologique de Kinshasa (JZK), qui ont changé leurs objectifs juridiques et économiques par une spoliation, sous l'œil impuissant des autorités politico-administratives de la ville province de Kinshasa. Concernant le premier espace vert, il a été créé pour une pépinière et en vue de conserver des espèces d'arbres rares pour une visite payante. Actuellement, le jardin botanique de Kinshasa a été transformé en un lieu de funérailles des personnalités tant politiques qu'artistes et autres. Pour les hommes politiques, à la mort d'un des leurs, ceux du pourvoir comme ceux de l'opposition se rencontrent pour partager leur douleur ; d'autres en profitent pour le pardon mutuel, d'autres encore viennent pour des transactions pour un poste politique ou pour faire voir à leurs électeurs la compassion qu'ils ont envers leurs collègues, ou encore tester leur popularité. S'agissant des artistes musiciens, les funérailles d'un des leur, restent un moyen de faire voir les modèles d'habits étrangers, de souliers, de voitures, etc. Et souvent, l'arrivée des musiciens vedettes crée des spectacles et drainent plusieurs mélomanes que l'on apprécie ou que l'on dénigre. Et cela crée aussi des polémiques entre les mélomanes. Les voyous, les fumeurs du chanvre, ... profitent de cette situation pour exhiber leurs talents dans le mal. En revanche, au jardin zoologique de Kinshasa, les différentes espèces de bêtes, même, rares, faisaient la fierté de cet espace auprès des nombreux visiteurs. Cet espace est devenu un endroit aux portes béantes où toutes les allées sont occupées par des terrasses et des restaurants de fortune qui ferment leurs portes au petit matin, où les amoureux se donnent rendez-vous pour boire et manger. Cela pousse les clients tantôt à apprécier le service d'accueil, tantôt la nourriture, tantôt à faire des commentaires sur l'ambiance, etc. Un lieu favorable aux amoureux, aux rencontres amicales, aux retrouvailles, mais c'est aussi l'occasion de disputes pour les rivaux.

\section{Conclusion}

La fonction principale des parcs et des espaces verts dans les villes est d'assurer un cadre favorisant les activités récréatives et sociales. En outre, ces espaces ont une grande importance pour l'environnement physique, la biodiversité et la qualité de l'air. 
Compte tenu de l'intérêt accru pour les problèmes écologiques urbains, il est souhaitable que les parcs et les espaces verts se développent considérablement durant les prochaines décennies.

L'appauvrissement social et biologique de la vie dans de nombreux espaces verts invite la population à une poussée de conscience individuelle et collective. Certains de ces espaces sont parfois appelés "déserts verts». Pour éviter ce genre de situations, nous devons apprendre à coopérer avec la nature, et non à lutter contre elle.

Au cours des prochaines années, pour la ville de Kinshasa, le principal défi sera de faire en sorte que l'expansion des infrastructures vertes dans les communes s'effectue dans le cadre du développement durable, sans l'emploi de techniques néfastes pour l'homme et la nature. La réussite de la bonne gestion des espaces verts dépend de l'effort conjugué de tous pour une nature saine. L’État et les entités territoriales décentralisées ont un rôle très important à jouer dans ce registre.

En rapport avec le développement durable de l'espace vert, voici quelques recommandations : la modernisation de l'administration publique plus précisément du Ministère de l'environnement et de la conservation de la nature ; l'assurance d'une bonne gouvernance environnementale; l'amélioration des mécanismes d'élaboration et d'évaluation des politiques publiques; l'élaboration de nouveaux cadres institutionnels en matière d'environnement et conservation de la nature, d'une part, d'urbanisme et d'habitat, d'autre part ; greffer l'éducation environnementale à partir de l'éducation formelle par des méthodes efficaces pour une prise en charge de la conscience environnementale ; la formation des agents de services de la ceinture verte et de reboisement, selon les nouvelles méthodes modernes de l'assainissement des espaces verts ; réhabilitation des espaces verts existants et renforcement en équipements modernes du service de la ceinture verte et de reboisement, des moyens et techniques matériels et financiers efficaces ; la sensibilisation, par les médias, la télévision, les théâtres, les randonnées, sur le bien-fondé des espaces verts artificiels et naturels ; la réglementation des lois, l'actualisation des textes sur les espaces verts et l'application des sanctions et amendes à l'encontre des contrevenants, l'application du principe pollueur-payeur; la nécessité de travailler en partenariat entre la commune (l'État) et les privés (les ONG, les bailleurs de fonds, ...) pour la gestion des espaces ; la nécessité que les espaces verts soient gérés par la commune où ils sont érigés et non par la mairie (recours à la décentralisation des services et de compétence) ; la population bénéficiaire des espaces verts doit avoir un comportement responsable vis-à-vis de l'environnement en général et des espaces verts en particulier ; prendre 
conscience envers l'environnement en général et les espaces verts en particulier ; utiliser à bon escient les espaces publics ; savoir que la gestion des espaces verts ne doit être le monopole ni des écologues, ni des écologistes, ni d'un parti politique, etc., mais que c'est l'affaire de tous. Elle implique une mixture gouvernants-gouvernés.

\section{Notes}

1. L'agora, est une place publique, chez les Grecs anciens, qui servait pour le marché et pour certains actes civils et politiques. Source : agora d'Athènes.(http://definition.ptidico.com/agora.htm ) 02/01/2009.

2. Lire à ce sujet Kaplan, R. et Kaplan, S., 1989, The Experience of Nature, Cambridge, Cambridge University Press.

3. Lire à ce sujet Ulrich, R.S., Simon, R.F., Losito, B.D., Fiorito E., Miles, MA., and Zetson, M., Stress Recovery during Exposure to Natural and Urban Environmental Psychology.

4. Lire à ce sujet Owen, J., 1992, The Ecology of a Garden, Cambridge,Cambridge University Press.

5. La tentative des Kinois de rebaptiser et de se réapproprier leur ville d'une façon négative illustre bien les essais de maîtrise permanente de la ville, en produisant des symboles de rejet, en définissant les places et en l'encapsulant dans le langage imagé.

6. Lire à ce sujet Hardoy, J., and Satiewaite, D., 1992, "Environmental Problems in the Third World Cities", in International Institute for Environment and Development, London.

7. En 1975, le président Mobutu, lors de son discours devant la Nation, demanda au peuple zaïrois de suivre l'exemple des Chinois qui ont développé leur pays grâce à la mécanisation de l'agriculture. A ce sujet, il demanda au peuple de « retrousser les manches ", en d'autres termes, de retourner à la terre.

8. Nous allons citer quelques ordonnances et décisions en rapport avec la gestion des espaces verts : - Piron P. et Devos J., 1959: 532-533, Codes et lois du Congo belge, T3. L'ord. du 28 juin 1959 relative à l'hygiène publique dans les agglomérations, dans son art.2, stipule de supprimer toute végétation susceptible de servir de gîte des déchets urbains ou autres le long des routes, des ronds- points, des jardins, des espaces de récréations. Art. 6 : Les contrevenants aux dispositions de cette ord. sont assortis d'une peine de servitude pénale de 2 ans au maximum et une amende qui ne dépassera pas 2000 francs ou d'une de ces peines seulement. Ord. n 62/181 du 25 juillet 1975 fixant le cadre organique des services publics de l'État et les conditions d'hygiène et d'assainissement des espaces verts en République du Zaïre (RDCongo); Arrêtén ${ }^{\circ} 15$ SC/0034/BGV/COJU/CM/98 du 17 février 1998 portant mesure des renforcements d'assainissement des espaces verts le long des boulevards et protection de la salubrité publique dans la ville de Kinshasa ; Ord. $n^{\circ} 127 / 6$ du 17juin 1980, art. 17 interdisant aux chefs coutumiers d'octroyer 
le permis de construction dans les espaces verts de Kinkole, Mitendi et Mbenseke Futi ; Art. 24. Interdisant l'abattage des arbres dans les espaces verts de Kinkole, Mitendi et Mbenseke Futi, etc.

9. Art. 18 de l'ordonnance $n^{\circ} 41 / 48$ du 12 février 1953.

10. Art. 10 de l'ordonnance $n^{\circ} 74 / 345$ du 28 juin 1959 précité ; article 8 de l'arrêté nSC/BGV/COJU/CM/98 du 18 avril 1998.

11. Ord. $n^{\circ} 74 / 345$ du 28 juin 1959, complété par l’Arrêté nº SC/0034/BGV COJU/ CM/98 du 18 avril 1998.

12. Lors de l'émission de Jean-Pierre Leperse de la CFI « Questions pour un champion ", la question était de savoir quelle est la ville la plus sale du monde. La réponse à cette question était : la ville de Kinshasa. Bien que cette réponse ait provoqué des réactions dans les médias congolais, elle reste une réalité. Lire ses auteurs pour confirmer les propos de Jean-Pierre Leperse : Pain Marc, 1984, Kinshasa : la ville et la cité, Paris, éd. De l’Orton collection mémoire, Munene Yamba-Yamba, «La problématique de la gestion des déchets à Kinshasa : aspects normatifs et institutionnels », in, Actes du $1^{\mathrm{e}}$ colloque sur la problématique des déchets à Kinshasa (Congo), Kinshasa du 12 au 15 août 1998 ; Kassay Ngur-Ikone Jules, 2008, « L'œil impuissant de l’Hôtel de ville de Kinshasa dans la prolifération des déchets en matière plastiques, » in LASK, Kinshasa, $n^{\circ}$ 52, pp.45-53, et tant d'autres.

\section{Bibliographie}

Actes du colloque de Lomé, 1997, « Les espaces verts à Lomé au cours de la période allemande », du 3-6 mars. Prospects: The 2005 Revision Population Database, Population Division, New York.

Arantxa, J., 2000, « Aménagement cyclable et espace urbain », in Association Métropolis.

Birou, A., 1997, « Appropriation de l'espace et pouvoirs dominants », Cahiers Options Méditerranéennes - CIHEAM, Vol. 23, 21-30.

Brunet, 2001, L'éthique dans la société de l'information, Québec et Paris, Presses de l’Université Laval et Harmattan.

Bureau d'Études d'Aménagement et d’Urbanisme (BEAU), 1985, Projet de développement urbain de Kinshasa (PDU).

Bureau d'Etudes et d'Aménagement Urbain (BEAU), 1990, Rapport annuel.

Bureau d'Etudes et d'Aménagement Urbain, 2000, Rapport annuel.

Catherine, R., 1982, “World Urbanization Prospects: The 2005 Revision Population Database”, Population Division, New York.

Centre d'Adaptation des Techniques Energie-Bois, 2000, Rapport annuel.

CIAM, 1959, La Charte d'Athènes en matière d'environnement, éd. de Minuit, p. 18.

Échos de la capitale Kinshasa, Edition n 3534.

Falloux, F., et Talbott, L., 1995, Environnement et développement, crise et opportunité, Paris, éd. Maison Neuve et la Rose.

Fare et Claterrbuck, Pourquoi et comment rendre sa ville plus verte, (www.entreprisedupaysage.org), 30 juillet 2006. 
Fraser, N., 1999, Rethinking the Public Sphere: A Contribution to the Critique of Actually Existing Democracy, in Craig Calhoun (Hg.), Habermas and the Public Sphere, Cambridge, pp. 109-142.

Georges, P., 1961, Précis de géographie urbaine, Paris, PUF.

Grahn, P., Attupplevaparken, Parken betydelse för alder, sluka och handikappade skildrede ghonom dagböcker, interjuer, teckninger och fotografier. -sveriges lantbruksuniversitet, Alnarp (en suédois).

Kanoepfel, Larrue et Varone, cités par Nahrath, S., 2005, Conférence sur « La politique publique : cadrage théorique », Lausanne, IDHEAP.

Kaplan, R., Kaplan, S., 1989, The Experience of Nature, Cambridge, Cambridge University Press.

Hardoy, J., Satterwaite, D., 1992, "Environmental Problems in the Third World Cities", in International Institute for Environment and Development, London.

Leimdorfer, F., 1999, « Enjeux et imaginaires de l'espace public à Abidjan. Discours d'acteurs », Politique Africaine, $\mathrm{n}^{\circ} 74$.

Le Potentiel, 2005, « Une croissance rapide pour la population de Kinshasa », in Echos de la capitale Kinshasa, Edition $n^{\circ} 3534$.

Maurel et Brunais, 1965, La conquête de la couleur, Paris, éd. Denoël.

Minte, M., et Malon, N., 2000, « L'espace vert à la ville de Bandundu : bilan et perspectives d'avenirs ", in Journal KIMPANGI, n 265.

Musinbondo Eyul'anki, D., 2008, « Du marasme d’un État-squelette au défi du développement durable. Gestion de l'environnement au Congo-Kinshasa : cueillette chronique et pauvreté durable », Chaire UNESCO, Université de Kinshasa.

Owen, J., 1992, The Ecology of a Garden, Cambridge, Cambridge University Press.

Pain Marc, 1984, Kinshasa : la ville et la cité, Paris, éd. De l’Orton collection mémoire.

Rfeitffer, E., 1966, Visage de la terre, Paris, PUF.

Schéma Directeur d'Aménagement et d'Urbanisme (SDAU), 1975.

Simon, J., 1952, L'art de connaître les arbres, Paris, PUF.

Soullier, L., 1968, Espaces verts et urbanisme, Paris, éd. Centre de Recherche d'Urbanisme, p. 50.United Nations, 2007, « World Urbanization Prospects: The 2005 Revision Population Database », Population Division, New York».

Ulrich, R.S., Simon, R.F., Losito, B.D., Fiorito E., Miles, M A., and Zetson, M., Stress Recovery during Exposure to Natural and Urban Environmental Psychology.

United Nations, 2007, «World Urbanization Prospects: The 2005 Revision Population Database », Population Division, New York.

Vitron, L., 1965, Dynamismes sociaux, Paris, éd. Ouvrières. 